\title{
ANALGÉSIE PÉRIDURALE À DOSE ÉlEVÉE DE FENTANYL: ÉCHEC DE LA MÉTHODE POUR LA KINÉSITHÉRAPIE POST-OPÉRATOIRE PRÉCOCE AVEC CHIRURGIE DU GENOU
}

\author{
M. Pierrot, M. Blaise, A. Dupuy, S. Hugon et M. Cupa
}

RESUME

\begin{abstract}
En post-opératoire immédiat de la chirurgie orthopédique du membre inférieur, 10 patients reçoivent fentanyl $5 \mu \mathrm{g} \cdot \mathrm{kg}^{-1}$ en une seule injection péridurale. L'analgésie quasi complète $(\mathrm{P}<0.001)$ est très rapidement obtenue. La période d'analgésie maxima est courte ( $87 \pm$ $8.34 \mathrm{~min})$, ainsi que la durée totale d'analgésie $(182.3 \pm 32.1 \mathrm{~min})$. Malgré cette dose élevée de fentanyl ( 245 à $450 \mu \mathrm{g}$ ), chez cinq patients, la mobilisation passive du genou après libération de l'appareil extenseur est extrêmement douloureuse, voire impossible chez trois d'entre eux, sans recourir aux anesthésiques locaux. A cette dose, le risque de dépression respiratoire est réel avec diminution de la fréquence respiratoire $(\mathrm{P}<0.01)$ et augmentation de la $\mathrm{PCO}_{2}(\mathrm{P}<0.01)$.

En regard de la courte durée de l'analgésie, d'un score analgésique identique aux études ayant utilisées de plus faibles doses de fentanyl ou d'autres morphinö̈des de plus longue duréc d'analgésic, de l'incapacité à calmer des douleurs provoquées intenses, et du risque réel de dépression respiratoirc, l'injection péridurale de fentanyl ne doit pas se faire à doses élevées, si tant est que son emploi soit préférable à celui de la morphine.
\end{abstract}

Mot Clefs: Analgesie, post-opératoire, péridurale, fentanyl; Complications, dépression respiratoire.

L'EXPÉRIMENTATION SUR l'animal ${ }^{1-4}$ et l'identification des récepteurs opioïdes, ${ }^{5,6}$ entre autres situés dans la substance gélatineuse de la corne dorsale de la moelle, ${ }^{7}$ justifient l'utilisation des morphinomimétiques par voie péridurale. ${ }^{8,9}$

Par voie parentérale, la puissance et la briéveté d'action du fentanyl en font un analgésique de choix en anesthésiologie. ${ }^{10}$ En regard de ces deux propriétés, les indications différentielles de son administration péridurale, par rapport aux autres morphinomimétiques ne sont pas énoncées dans la littérature. ${ }^{11}$

Ce travail se propose d'évaluer, d'une part en post-opératoire, l'efficacité analgésique d'une dose élevée de fentanyl injectée par voie péridurale, pour effectuer une kinésithérapie particulièrement douloureuse; d'autre part, les effets indésirables d'une telle posologie.

\section{Matériel et Méthodes}

Dix patients, sept hommes et trois femmes, sans antécédent cardiovasculaire ni respiratoire, agés en moyenne de $52.9 \pm 15.8$ ans (35-83

M. Pierrot, Chef de Clinique Assistant, Département d'Anesthésie-Réanimation, Hôpital Avicenne, 125, route de Stalingrad, 93009, Bobigny, France. ans) et pesant $68.1 \pm 13 \mathrm{~kg}(49-90)$, sont anesthêsiés au niveau des membres inférieurs par voie péridurale, pour qu'il leur soit effectué une intervention de chirurgie orthopédique (Tableau I).

La ponction péridurale est réalisée dans l'espace L3 L4 par la méthode du mandrin liquide. Après mise en place d'un cathéter l'anesthésie est obtenue par une solution composée à mipartie de chlorhydrate de lidocaine à 2 pour cent adrénalinée* et de chlorhydrate de bupivacaïne à 0.5 pour cent $\dagger$. Elle autorise alors la libération de l'appareil extenseur du genou (LAEG) chez cinq patients, chez trois autres la réparation d'entorses graves du genou (EG) et chez deux autres une chirurgie réparatrice du pied.

En salle de éveil, pendant la première journée postopératoire, les réinjections de bupivicaïne à 0.5 pour cent vérifient le maintien du cathéter péridural. Après accord de chacun des patients, à la 24ème heure, le fentanyl $\ddagger$ seul (sans excipient) est injecté à la dose unique de $5 \mu \mathrm{g} \cdot \mathrm{kg}^{-1}$. lon).

${ }^{*}$ Chlorhydrate de Lidocaïne: Xylocaïne (R. Belton).

Chlothydrate de Bupivacaine: Marcaïne (Bofort Nobel Pharma).

$\ddagger$ Citrate de Fentanyl: (Janssen-Lebrun).

Can. Anaesth. Soc. J., vol. 29, no. 6, November 1982 
TABLEAU I

CONDITIONS DE L'ÉTUde LAEG: LIBÉRATION DE L'APPAREIL

Extérieur du Genou. E.G.: Entorse Grave du Genou. Pied: Chirurgie Reparatrice du Pied

\begin{tabular}{rccccc}
\hline \hline $\mathrm{N}^{\circ}$ & Sexe & Age & Poids/Kg & $\begin{array}{c}\text { Dose } \\
\text { Fentanyl } / \mu \mathrm{g}\end{array}$ & $\begin{array}{c}\text { Type de } \\
\text { Chirurgie }\end{array}$ \\
\hline $\mathrm{l}$ & $\mathrm{M}$ & 35 & 75 & 375 & LAEG \\
2 & $\mathrm{M}$ & 42 & 68 & 340 & LAEG \\
3 & $\mathrm{M}$ & 45 & 54 & 270 & LAEG \\
4 & $\mathrm{M}$ & 56 & 90 & 450 & LAEG \\
5 & $\mathrm{~F}$ & 38 & 65 & 325 & LAEG \\
6 & $\mathrm{M}$ & 61 & 71 & 355 & EG \\
7 & $\mathrm{~F}$ & 59 & 63 & 315 & EG \\
8 & $\mathrm{M}$ & 71 & 88 & 440 & EG \\
9 & $\mathrm{M}$ & 39 & 58 & 290 & Pied \\
10 & $\mathrm{~F}$ & 83 & 49 & 245 & Pied \\
\hline $\mathrm{n}=10$ & $7 \mathrm{M}$ & 52.9 & 68.1 & 340.5 & \\
& $3 \mathrm{~F}$ & \pm 15.8 & \pm 13.47 & \pm 67.4 & \\
\hline
\end{tabular}

Le résultat analgésique est quantifié de 0 à 10 par la méthode de l'échelle visuelle: elle utilise une bande de papier de $10 \mathrm{~cm}$ dont chacune des extrémités représente respectivement l'absence totale, et le maximum de douleur. La marque faite par le patient, expression de sa sensation douloureuse est ensuite mesurée en centimètres, à son insu.

Trente à 60 minutes après l'injection de fentanyI, la mobilisation passive du genou des cinq patients "LAEG" est effectuée.

Parallèlement à cette étude de l'analgésie, au maximum d'intensité de celle ci, nous relevons les modifications de la conscience et des pupilles, les variations de la fréquence cardiaque et de I'E.C.G. (Appareil HP 78342 A), et de la pression artérielle systémique (Dynamap). Du point de vue ventilatoire, la fréquence respiratoire (FR) est mesurée ainsi que le volume courant (Vt) (Spiromètre Drager 28083). Les gaz du sang sont prélevés au niveau de l'artère radiale.

A l'aide d'une calculatrice (HP $41 \mathrm{C}$ ), le traitement statistique est effectué par le T-test de Student apparié dont la significativité est extraite des Tables de Fisher et Yates.

\section{RÉsultats}

Cinq patients suspects de brèches méningées ou vasculaires ont été exclus de l'étude qui intéresse donc 10 patients.

L'anesthésie péridurale nécessite en moyenne l'injection de $13 \pm 4 \mathrm{ml}$ de lidocaïne à deux pour cent et de $22 \pm 5 \mathrm{ml}$ de bupivacaïne. L'analgésie péridurale post-opératoire-s'effectue par une dose moyenne de $340.5 \pm 67.4 \mu \mathrm{g}$ (extrêmes: $245-450 \mu \mathrm{g}$ ) de fentanyl.

Tableau II. L'ensemble de 10 patients présente au repos une douleur intense, cotée à 7.39 \pm 1.49 en moyenne. Cependant, le score apparait significativement plus élevé $(P<0.01)$ dans le groupe opéré pour LAEG $(8.52 \pm 0.78)$ que chez le reste des patients $(6.3 \pm 0.7)$. L'injection péridurale de fentanyl réduit cette douleur de repos $(P<0.001)$. Cette analgésie quasi complète $(0.64 \pm 0.22)$ s'installe $30 \mathrm{~min}$ après l'injection et s'accompagne d'une sensation de bien-être régulièrement signalée par les patients. Dès la 5ème minute, elle est précédée d'une hypoalgésie. La douleur réapparait en $87 \pm 8.3$ min; son intensité initiale est restituée en 182.3

TABLEAU II

INTENSITE DE LA DOULEUR AVANT ET 30 Min Après L'injection Péridurale de FENTANYL

\begin{tabular}{rcc}
\hline \hline $\mathrm{N}^{\mathrm{e}}$ & Avant Fentanyl & Après Fentanyl \\
\hline 1 & 9.5 & 0.5 \\
2 & 8.2 & 0.8 \\
3 & 8.7 & 1.2 \\
4 & 7.2 & 0.6 \\
5 & 8.8 & 0.5 \\
6 & 7.4 & 0.7 \\
7 & 6.2 & 0.5 \\
8 & 5.8 & 0.5 \\
9 & 7.4 & 0.5 \\
10 & 4.7 & 0.6 \\
\hline $\mathrm{n}=10$ & 7.39 & 0.64 \\
& \pm 1.49 & \pm 0.22 \\
\hline \multicolumn{3}{c}{} \\
\hline
\end{tabular}


TABLEAU III

Chronologie de L'ANalgésie

\begin{tabular}{|c|c|c|c|c|c|}
\hline \multirow[b]{2}{*}{$\mathrm{N}^{\circ}$} & \multirow{2}{*}{$\begin{array}{c}\text { Fentanyl } \\
\mu \mathrm{g}\end{array}$} & \multirow{2}{*}{$\begin{array}{l}\text { Hypoalgésie } \\
\text { Latence/min }\end{array}$} & \multicolumn{2}{|c|}{$\begin{array}{c}\text { Analgésic Maximalc } \\
\text { de Repos }\end{array}$} & \multirow{2}{*}{$\begin{array}{c}\text { Analgésie } \\
\text { Duréc Totale } \\
\text { min }\end{array}$} \\
\hline & & & Latence min & Durée min & \\
\hline 1 & 375 & 7 & 26 & 84 & 120 \\
\hline 2 & 340 & 4 & 28 & 92 & 230 \\
\hline 3 & 270 & 5 & 35 & 72 & 170 \\
\hline 4 & 450 & 5 & 25 & 98 & 184 \\
\hline 5 & 325 & 6 & 32 & 95 & 150 \\
\hline 6 & 355 & 3 & 27 & 78 & 212 \\
\hline 7 & 315 & 8 & 40 & 91 & 195 \\
\hline 8 & 440 & 5 & 25 & 87 & 166 \\
\hline 9 & 290 & 6 & 25 & 80 & 200 \\
\hline 10 & 245 & 4 & 34 & 93 & 192 \\
\hline$n=10$ & $\begin{array}{r}340.5 \\
\pm 67.4\end{array}$ & $\begin{aligned} & 5.3 \\
\pm & 1.49\end{aligned}$ & $\begin{array}{l}30.1 \\
\pm 5.25\end{array}$ & $\begin{array}{l}87 \\
\pm 8.34\end{array}$ & $\begin{array}{r}182.30 \\
\pm 32.14\end{array}$ \\
\hline
\end{tabular}

TABLEAU IV

Evolution des Paramètres Ventilatoires Avant (AF) et 30 min après (F) L'injection Péridurale DU FENTANYL

\begin{tabular}{|c|c|c|c|c|c|c|c|c|}
\hline \multirow[b]{2}{*}{$\mathbf{N}^{\circ}$} & \multicolumn{2}{|c|}{ FR/MIN } & \multicolumn{2}{|c|}{ VT/ML } & \multicolumn{2}{|c|}{$\mathrm{Po}_{2}(\mathrm{kPa})$} & \multicolumn{2}{|c|}{$\mathrm{PCO}_{2}(\mathrm{kPa})$} \\
\hline & AF & $\mathrm{F}$ & $\mathrm{AF}$ & $\mathrm{F}$ & $\mathrm{AF}$ & $\mathbf{F}$ & AF & $\mathrm{F}$ \\
\hline 1 & 18 & 16 & 600 & 530 & 10.6 & 10.14 & 5.07 & 5.07 \\
\hline 2 & $\begin{array}{l}10 \\
24\end{array}$ & $\begin{array}{l}10 \\
15\end{array}$ & 520 & 500 & 10.1 & 10.4 & 5.2 & 5.2 \\
\hline 3 & $\begin{array}{l}24 \\
17\end{array}$ & 11 & 510 & 600 & 11.8 & 11.5 & 4.55 & 4.94 \\
\hline 4 & 20 & 13 & 680 & 475 & 11.05 & 10.66 & 5.46 & 5.2 \\
\hline 5 & 26 & 18 & 640 & 620 & 8.4 & 9.36 & 4.29 & 5.07 \\
\hline 6 & 20 & 20 & 540 & 570 & 11.4 & 9.36 & 4.94 & 5.72 \\
\hline 7 & 23 & 16 & 700 & 580 & 11.9 & 9.23 & 4.03 & 5.2 \\
\hline 8 & 27 & 6 & 670 & 300 & 9.4 & 7.8 & 5.72 & 6.24 \\
\hline 9 & 21 & 12 & 450 & 450 & 9.7 & 8.97 & 4.03 & 4.55 \\
\hline 10 & 19 & 15 & 610 & 540 & 11.4 & 10.79 & 4.68 & 4.99 \\
\hline \multirow[t]{2}{*}{$n=10$} & 21.5 & $\begin{array}{c}14.2 \\
+394\end{array}$ & $\begin{array}{c}592 \\
+836\end{array}$ & $\begin{array}{r}516.5 \\
+934\end{array}$ & $\begin{array}{r}10.5 \\
+1.15\end{array}$ & $\begin{array}{r}9.78 \\
+101\end{array}$ & $\begin{array}{r}4.8 \\
+0.58\end{array}$ & $\begin{array}{r}5.22 \\
+046\end{array}$ \\
\hline & \multicolumn{2}{|c|}{$P<0.01$} & \multicolumn{2}{|c|}{ NS } & \multicolumn{2}{|c|}{ NS } & \multicolumn{2}{|c|}{$P<0.01$} \\
\hline
\end{tabular}

$\pm 32.1 \mathrm{~min}$ (Tableau III). Aucune de ces durées n'est corrélée à la dose de fentanyl injectée.

Au maximum de l'analgésie, entre la 30ème et 60ème minute après LAEG, la mise en flexion passive, progressive, du genou, provoque constamment chez les cing patients une douleur intense, qualifiée d'insupportable par trois d'entre eux. La mobilisation achevée, ou arrêtée, sans autre injection de fentanyl, l'analgésie quasi complète est restituée en $5 \pm 3 \mathrm{~min}$; au terme desquelles on retrouve un score identique à celui précédent la manipulation. Devant l'échec total de l'analgésie par le fentanyl, les autres mobilisations sont ensuite effectuées après injection de lidocaïne, par le cathéter péridural.

L'injection péridurale de fentanyl provoque aussi des effets indésirables: pendant l'injection, la sensation de brulure est notée huit fois sur dix; au maximum de l'analgésie, tous les patients présentent un myosis et une légère somnolence, celle-ci n'empêche jamais les réponses coordonnées aux stimuli verbaux; les paramètres hémodynamiques périphériques, pouls et pression artérielle n'accusent aucune variation. Par contre (Tableau IV) la fréquence respiratoire s'abaisse constamment, en moyenne de $21.5 \pm$ $3.37 / \mathrm{min}$ à $14.2 \pm 2.8 / \mathrm{min}(\mathrm{P}<0.001)$. Le 
volume courant ne diminue pas de façon significative; cette diminution de la ventilation, progressive et retardée par rapport à l'hypoalgésie, se stabilise à la 30ème minute. Elle persiste pendant toute la durée de l'analgésie maximale avec variation significative de la $\mathrm{PCO}_{2}(\mathrm{P}<$ 0.01 ). Seul le patient $\mathrm{n}^{\circ} 8$ présente une bradypnée avec hypoventilation $\left(\mathrm{Po}_{2} 8 \mathrm{kPa}\right.$ : $\mathrm{PCO}_{2} 6.24 \mathrm{kPa}$ ) corrigée par les incitations verbales et surtout l'injection intraveineuse de naloxone $(0.8 \mathrm{mg})$.

\section{Discussion}

L'analgésie induite par l'injection péridurale de morphinomimétiques est désormais couramment employée dans de nombreuses situations. ${ }^{12}$ En post-opératoire, la littérature applique cette technique aux douleurs de repos après chirurgie viscérale, abdominale ou pelvienne. ${ }^{12-16}$ Deux raisons nous ont fait préférer l'étude de l'analgésie péridurale, après intervention orthopédique du membre inférieur: (a) Cette dernière est conduite sans anesthésie générale, sans analgésique I.V. ou I.M. (b) La LAEG est marquée par un syndrome douloureux postopératoire intense qui doit être résolu pour pratiquer un kinćsithérapie indispensable.

Quantifier cliniquement l'intensité douloureuse et l'analgésie reste un problème délicat. ${ }^{17}$ Avec d'autres ${ }^{9,16,18-20}$ nous avons retenu la méthode utilisée par Scott et Huskisson ${ }^{21}$ et validee par Revill, et al. ${ }^{22}$ Elle préconise l'emploi d'une échelle linéaire d'au moins $10 \mathrm{~cm}$ entre les extrémités de laquelle le patient situe l'intensité de sa douleur. La mesure de cet index témoigne d'une mémorisation stable dans le temps, des phénomènes douloureux que ne modifient pas les variations psycho-sensorielles provoquées par la meperidine.

La morphine est le morphinoïde le plus fréquemment injecté dans l'espace péridural. Contre les douleurs de repos, chroniques et cancéreuses, ${ }^{9,18,23,24}$ ou subaigues et postopératoires, elle donne des résultats constamment satisfaisants, même à faible dose, et donc, au prix d'effet indésirable, acceptable.

Le seule utilisation de morphine ne permet pas de pratiquer d'acte de chirurgie. ${ }^{15,25}$ Les douleurs intenses de l'obstétrique sont inconstamment résolues par l'injection péridurale de morphine ${ }^{12,26-29}$ ainsi que celle de l'immédiat postopératoire.$^{30} \mathrm{~A}$ ce jour, l'utilisation péridurale de fentanyl, de plus grande puissance analgésique par voie I.V., est peu fréquente ${ }^{19,29,31-33}$ : elle pose plus de questions qu'elle n'apporte de réponses.

Wolfe ${ }^{33}$ Lomessy, ${ }^{19}$ utilisent respectivement 100 et $200 \mu \mathrm{g}$ de fentanyl alors que nous injections $5 \mu \mathrm{g} \cdot \mathrm{kg}^{-1}$, soit en moyenne $340 \mu \mathrm{g}$. La douleur de repos de nos patients, notamment ceux opérés pour LAEG, est plus élevée que dans l'article de Lomessy, nous obtenons cependant un résultat analgésique identique. La dose dépendance de l'intensité analgésique semble unanimement reconnu. ${ }^{34}$ Cependant, les doses effectivement élevées que nous employons ne permettent jamais une kinésithérapie postopératoire précoce, indolore; celle-ci est, sans comparaison, obtenue grace aux anesthésiques locaux. Aussi, plutôt qu'insuffisante, l'analgésie du fentanyl en péridurale nous parait totalement inadéquat au but que nous nous êtions fixé.

L'analgésie débute aussi entre la 5ème et 10ème minute et atteint son maximum entre la 22 ème et 38 ème minute. Cette période de latence n'est donc pas plus courte que celle notée avec la morphine ${ }^{19}$ ou la meperidine. ${ }^{13}$ La phase d'analgésie maximale est courte, de l'ordre de 90 minutes dans nos observations comme dans celles de McQuay. ${ }^{32}$ La durée maximale d'analgésie dure $182 \pm 32 \mathrm{~min}$; elle est donc proche de celle notée par Wolfe (200 à 240 min). Les longues durées d'analgésie (240 à $600 \mathrm{~min}$ ) observées par Lomessy ${ }^{19}$ sont donc surprenantes et peut-être pas sans rapport avec l'anesthésie générale préalable. La dose dépendance de la durée de l'analgésie, expérimentalement retrouvée avec la morphine, ${ }^{35}$ n'est pas ici établi pour le fentanyl. D'ailleurs, pour de faibles doses de morphine, de 0.5 à $\mathrm{l}$ mg, Wang ${ }^{9}$ note des analgésies prolongées de 15 à $24 \mathrm{~h}$. De son côté avec $20 \mathrm{mg}$, Samii ${ }^{36} n^{\prime}$ 'accroit pas de façon majeure cette analgésie ( $27 \pm 2$ h.). Avec la meperidine, Cousins ${ }^{13}$ montre des durées moyennes d'analgésies de $8 \mathrm{~h}$. avec $30 \mathrm{mg}$ et de $6 \mathrm{~h}$. avec $100 \mathrm{mg}$; à cette dose, l'analgésie résulterait d'une action spinale à travers la dure mère et d'une action centrale dépendante de taux sanguin de meperidine. Certe dernière corrélation n'est pas retrouvée par Weddel avec la morphine ${ }^{20}$ Ainsi, du point de vue de la durée, avec Perriss, ${ }^{31}$ l'emploi du fentanyl n'améliore pas la technique d'analgésie péridurale par les seules morphinoïdes.

Le myosis et la somnolence, au maximum de l'analgésie, témoignent d'une imprégnation supra-spinal, ${ }^{37}$ par le fentanyl, qui ne permet pas d'attribuer à la seule analgésie la diminution de la frequence respiratoire, dramatique chez une 
de nos patientes. La dépression respiratoire relevée après injection de morphine et de meperidine, intrathécale ou péridurale, ${ }^{38-41}$ n'avait semble-t-il pas été notée jusqu'à présent avec le fentanyl ${ }^{42}$; la grande liposolubilité du fentanyl, ${ }^{43}$ sa faible diffusion plasmatique à partir du $\mathrm{LCR}^{33}$ et une action à peine plus puissante que la morphine dans ce milieu ${ }^{43}$ en seraient les raisons. Notre étude réalisée sur des sujets en décubitus et surtout à plus fortes doses témoigne d'un risque réel de dépression respiratoire, probablement dose-dépendante comme avec toutes les substances de cette classe. ${ }^{34}$

Dès lors, i] ne nous semble pas que l'utilisation du fentanyl seul en péridurale puisse apporter un bénéfice par rapport à la morphine: a) sa puissance par voie systémique n'est pas ici retrouvée et notamment elle ne permet pas d'effectuer des actes très douloureux; $b$ ) sa durée d'action, nettement plus courte ne s'allonge pas avec l'augmentation de la posologie; c) ce qui est par contre susceptible de provoquer une dépression respiratoire. A ce prix ${ }^{34}$ l'emploi du fentanyl en péridurale ne nous semble pas justifiable.

\section{REMERCIEMENT}

Les auteurs remercient Melle Rollin F. pour l'aide apportée à la rédaction de ce travail.

\section{RÉFÉRENCES}

1. Besson, J.M., Wyon-MaIllard, J.M., Benoist, C., Conseiller, C. \& Hamann, K.F. Effects of phenoperidine on lamina $V$ cells in the cat dorsal horn. J. Pharmacol. Exp. Therap. 187: 239/245 (1973)

2. Kitahata, L.M., Kosaka, Y., Taub, A., BonIKos, K. \& Hoffer, M. Lamina specific suppression of dorsal horn unit activity by morphine sulfate. Anesthesiology 41: 39/48 (1974)

3. Calvillo, O., Henry, J.L. \& Neuman, R.S. Effects of morphine and naloxone on dorsal horn neurones in the cat. Can. J. Physiol. Pharmacol. 52: 1207/1211 (1974)

4. Le Bars, D. Menetrey, D., Conseiller, C. \& Besson, J. Depressive effects of morphine upon lamina $V$ cells activities in the dorsal horn of the spinal cat. Brain Res. 98: 261/277 (1975).

5. Pert, C.B. \& SNyder, S.H. Opiate receptor demonstration in nervous tissue. Science 179 : $1011 / 1014$ (1973).

6. ATWEH, S.F. \& KUHAR, M.J. Autoradiographie localization of opiate receptors in rat brain. $I$. Spiral cord and lower medulla. Brain Res. 124: $53 / 67$ (1977).

7. Duggan, A.W., Hall, J.G. \& Headley, P.M. Suppression of transmission of nociceptives impulses by morphine: selective effects of morphine administered in the region of substantia gelatinosa. Br. J. Pharmacol. 61: 65/76 (1977).

8. YAKSH, T.L. \& RUDY, T.A. Analgesia mediated by a direct spinal action of narcotics. Science 192: $1357 / 1358$ (1976).

9. Wang, J.K. Nauss, L. A.\& Thomas, J.E. Pain relief by intrathecally applied morphine in man. Anesthesiology 50: 149/151 (1979).

10. Romagnoli, A. Duration of action of fentanyl. Anesthesiology 39: 568/569 (1973)

11. De Castro, D' Inverno, E., Lecron, L. Levy, D. \& Toppet-Balatoni. Perspectives d'utilisation de morphinö̈des en anesthésie locorégionale. Justification. Premiers résultats. Anesth. Analg. Rean. 37: 17/24 (1980).

12. Magora, F., Olshwang, D., Elmerl, D., Shork, J., Katzenelson, R., Cotev, S. \& DAVIDSON, J.T. Observation on extra-dural morphine analgesia in various pain conditions. $\mathrm{Br} . \mathrm{J}$. Anaesth. 52: 247/252 (1980)

13. Cousins, M.J., Mather, L.E., Glynn, C.J., Wilson, P.R. \& Graham, J.R. Selective spinal analgesia. Lancet 1: 1141/1142 (1979).

14. Bromage, P.R. Camporesi, E. \& Chestnut, D. Epidural narcotics for postoperative analgesia. Anesth. Analg. (Cleve) 59:473/480 (1980).

15. Graham, J.L., King, R. \& McCaughey, W. Postoperative pain relief using epidural morphine. Anaesthesia 35: 158/160 (1980).

16. Asari, H., Inoue, K., Shibata, T. \& Soga, T. Segmental effect of morphine injected into the epidural space in man. Anesthesiology 54: 75/77 (1981).

17. Sternbach, R.A., Murphy, R.W. \& TimmerMANS, $G$. Measuring the severity of clinical pain. Adv. Neurol. 4: $281 / 288$ (1974).

18. Coombs, D.W., Sauders, R.L., Gaylor, M.S., Pageau, M.G., L.eith, M.G. \& SchalBERGER, C. Continuous epidural analgesia via implanted morphine reservoir. Lancet $2: 425 / 426$ (1981).

19. Lomessy, A., Viale, J.P. \& Motin, J. Analgesie post-opératoire au fentanyl par voie péridurale. Ann. Anesth. Franc. I: 17/21 (1981)

20. WedDel, S.J. \& Ritter, R.R. Serum levels following epidural administration of morphine and correlation with relief of post surgical pain. Anesthesiology 54: $210 / 214$ (1981).

21. SCOTT, J. \& HUSKINSON, E.C. Graphic representation of pain. Pain 2: 175/184 (1976).

22. Revill, S.I., RoBinson, J.O., ROSEN, M. \& HoGg, M.I.J. The reliability of a linear analogue for evaluating pain. Anaesthesia 31: 1191/1198 (1976).

23. Bahar, M., Magora, F., Olshwang, D. \& DAVIDSON, J.T. Epidural morphine in treatment of pain. Lancet I: 527/528 (1979).

24. Zenz, M Schappler-Scheele, B. Neuhaus, R., Piepenbrock, S. \& Hilfrich, J. Long term peridural morphine analgesia in cancer pain. Lancet $1: 91$ (1981).

25. Muller, A., Laugner, B., Farcot, J.M., Singer, M., GAUTHIER-LAFAYE, P. \& GANDAR, R. Hypoalgésie obstétricale par injection péridurale de morphine. Anesth. Anal. Rean. 38: 35/41 (1981).

26. Husemeyer, R.P., O'ConNor, M.C. \& DAN- 
VENPORT, H. Failure of epidural morphine to relieve pain in labour. Anaesthesia 35: 161/163 (1980).

27. Booker, P.D., Wilkes, R.G. \& Beddard, J. Obstetric pain relief using epidural morphine. Anaesthesia 35: 377/379 (1980).

28. Perriss, B.W. Epidural pethidine in labour. A study of dose requirements. Anaesthesia 35 : $380 / 382$ (1980).

29. Carrie, L.E.S., O'Sullivan, G.M. \& SeegoBIN, R. Epidural fentanyl in labour (Forum). Anaesthesia 36: 965/969 (1981).

30. McClure, J.H., Chambers, W.A., Moore, E. \& ScotT, D.B. Epidural morphine for post operative pain. Lancet $1: 975 / 976(1980)$.

31. Perriss, B.W. Epidural opiates in labor. Lancet 2: 422 (1979)

32. McQuay, H.J., Bullingham, R.E.S., Evans, P.J.D., Lloyd, J.W. \& Moore, R.A. Demand analgesia to assess pain relief from epidural opiates. Lancet $I: 768 / 769$ (1980).

33. Wolfe, M.J. \& Davies, G.K. Analgesic action of extradural fentanyl. Brit. J. Anaesth. 52: 357/358 (1980)

34. Bromage, P.R. The price of intraspinal narcotic analgesia: Basic constraints. Anesth. Analg. (Clev.) 60: 461/463 (1981).

35. Yaksh, T.L., WILSON, P.R., Kaikor, F. \& INTURRISI, CH.E. Analgesia produces by a spinal action of morphine and effects upon paturition in the rat. Anesthesiology 51: 386/392 (1979).
36. Samit, K., Feret, J., Harart, A. \& Viars, P. Selective spinal analgesia. Lancet $I: 1142$ (1979).

37. BaHAR, M., OrR, I.A. \& DundeE, J.W. Shrinking pupils as a warning of respiratory depression after spinal morphine. Lancet $1: 893$ (1981).

38. Yagishita, Y., Fukuda, K. \& Moriok, T Intrathecal application of morphine: I. as a supplementation of anesthesia and a prolonged relief of post operative pain. Jpn. J. Anesth. 28: $1584 / 1591$ (1979).

39. SCOTT, D.D. \& MCClure, J. Selective epidural analgesia. Lancet 1: 1410/1411 (1979).

40. GlynN, C.J., Mather, LE., Cousins, M.J., WILSON, P.R. \& GrahaM, J.R. Spinal narcotics and respiratory depression. Lancet 2: 356/357 (1979).

41. Baskoff, J.D., Watson, R.L. \& Muldoon, SM. Respiratory arrest after intrathecal morphine. Anesthesiol. Rev. 7: 12/15 (1980).

42. WOLfe, M.J. \& NichOlas, A.D.G. Selective epidural analgesia. Lancet 2: 150/151 (1979).

43. Von Cube, B., Teschemacher, H., Herz, A. \& HEss, R. Permeation morphinartig wirksamer substanzen an den ort in abhängigheit von ihrer uporlöslickeit nach intraven öser applikation. Naunyn. Schmiedeberg Arch. Pharmak. 265: $455 / 473$ (1970).

\section{ABSTRACT}

Following orthopedic surgery of the lower limb, ten patients were given fentanyl $5 \mu \mathrm{g} \cdot \mathrm{kg}^{-1}$ in a single epidural injection. Almost complete analgesia $(P<0.001)$ was rapidly obtained. The total period of analgesia was rather short $(182.3 \pm 32.1 \mathrm{~min})$. The maximal analgesia period was $87 \pm 8.34$ minutes. Despite this high dose of fentanyl ( 245 to $450 \mu \mathrm{g}$ ), in five patients the passive mobilization of the knee following surgery was extremely painful and, for that matter, impossible in three of them. Such high doses of fentanyl entail the risk of respiratory depression as respiratory rate is decreased $(\mathrm{P}<0.01)$ and the $\mathrm{PCO}_{2}$ is increased $(\mathrm{P}<0.01)$.

Fentanyl should not be used at such high dosage and should probably not be preferred to morphine, considering that the duration of analgesia is short, that the analgesic scorc is identical to that obtained with lower doses or with longer lasting narcotics, that it docs not prevent passive mobilization pains and that it entails a definite risk of respiratory depression. 